\title{
Burning the Haystack to Save the Needle: Dimension Reduction and Privacy in Text and Network Data
}

\author{
Alexander Murray-Watters
}

\begin{abstract}
This poster presents results from applying a new dimension reduction technique (UMAP) to a wide variety of data types, ranging from online text to social networks, for the purpose of creating useful, but anonymized, data. As the dimension reduction procedure produces meaningful distances and supports arbitrary distance measures, it can be applied to a variety of problems, and produces data that is useful for both visualization and predictive modeling. Included is a description of the dimension reduction procedure, the results of its application, and a discussion of planned future use.
\end{abstract}

How can we increase the difficulty of recovering the identity of individuals in a dataset, while still being able to make use of the modified data? This problem is particularly difficult when dealing with online datasets, as easily searchable third-party information may be available, such as in the Netflix prize case, where researchers managed to use IMDB movie reviews to deanonymize individuals(Narayanan and Shmatikov 2006). Deanonymization becomes an even more difficult problem when social network data is taken into account, as even small changes to a social network (perhaps done to preserve anonymity) can have serious implications for analysis.

Previous attempts to ameliorate the problem of deanonymization, such as the use of synthetic data, focused on dealing with traditional rectangular datasets, rather than text or social network data. Synthetic data involves generating data that looks "similar" to the original data, but isn't identical. The method usually assumes a particular model, often only for a particular application - say, a specific linear regression. Other methods involve adding noise to the data, which is usually easier to reverse than might be expected, and distributing a correlation matrix instead of the raw data, which limits the kinds of variables that can be anonymized. One of the most promising methods for a general anonymization technique is $\epsilon$-differential privacy (which places a bound on the amount of privacy an individual would lose by participating), which unfortunately requires each individual is represented by a row in a dataset (Heffetz and Ligett 2014), inhibiting its use with both (social) network and text data.

\section{Proposed solution}

This poster applies a dimension reduction procedure that makes few assumptions and produces dimensions that have meaningful distances - UMAP (McInnes and Healy 2018). We can then use these new dimensions as anonymized data within various analyses, including supervised learning (regression). As this kind of dimension reduction is lossy (meaning it loses some information), the original data cannot be completely recovered from the dimension reduced data. This loss of data acts as an anonymization, in that some of the identifying information is lost during the reduction.

\section{A brief description of how UMAP works}

Roughly, the UMAP procedure creates a unique distance measure for every observation, calculates the distance between that observation and its $n$ nearest neighbors, then stitches the results together using "fuzzy simplicial sets" so that we can meaningfully talk about distance between the different points. For an explanation of some of the background concepts involved, see (Awodey 2010). The procedure then performs its dimension reduction on this stitched together space.

As the procedure's run time increases with the number of observations, not the number of variables, it is well suited to high-dimensional problems, which we are likely to observe in both text and social network datasets.

UMAP assumes:

1. Data are uniformly distributed on a Riemannian manifold (easiest to think of this as a Cartesian plane wrapped around another object - e.g., a sphere). This is a nasty assumption, but we can get around it.

2. Our Riemannian metric (how we calculate distance on the manifold) is locally constant (meaning, within a particular 'area' we can calculate distance the same way).

3. Our manifold is locally connected (meaning we don't have a disconnect between two sections of the manifold - e.g., someone hasn't cut our sphere into two separate pieces)

These are very weak assumptions as far as dimension reduction usually goes. It allows for non-linear relations, and doesn't assume a particular distribution - e.g., Gaussian. 


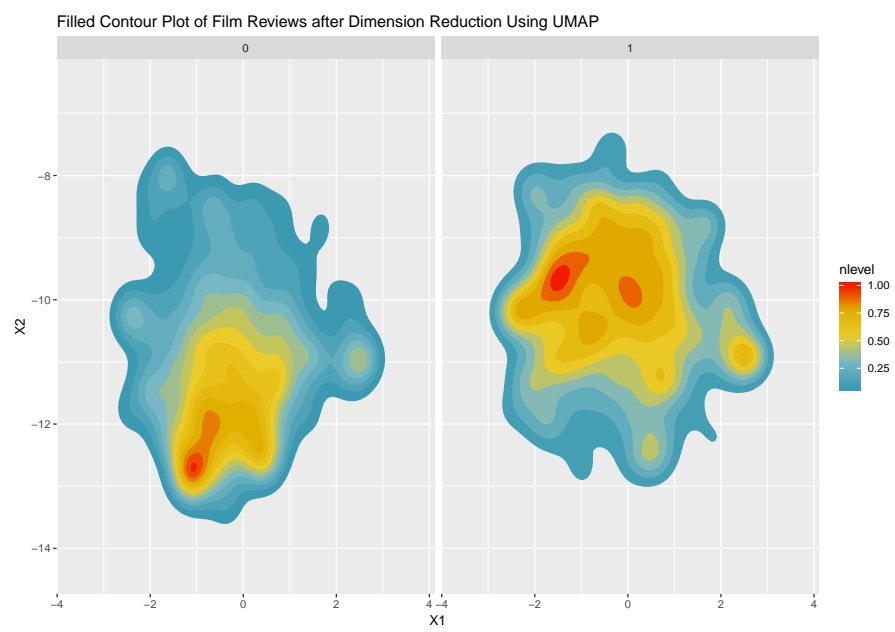

Figure 1: Result of performing dimension reduction on IMDB text data.

We can also use many different distance metrics (e.g., Euclidean, cosine distance, Hamming distance, etc), enabling us to apply the procedure to a variety of different kinds of data.

\section{An example of applying UMAP to text data}

We use the movie_review dataset from the text2vec package (Selivanov 2016), which contains 5000 reviews of films from IMDB, with a binary "sentiment" variable denoting film enjoyment, based off the numerical rating given (on a scale from 1 to 10). A 0 sentiment implies a rating less than 5 , while a 1 rating implies a rating greater than or equal to 7 .

Converting the reviews to a bag-of-words representation (i.e., a simple frequency count of each word in a review) and removing stopwords (e.g., the, and, of) using the stopwords function from the tm R package (Meyer, Hornik, and Feinerer 2008), yields a 5,000 row (review) by 7,522 column (word) matrix:

After using UMAP to reduce this large matrix to 2 dimensions ${ }^{1}$, we observe (Figure 1) that the dimension reduction has preserved some of the differences in text between the binary sentiment categories. This is clear, in that the two categories have peak densities located in different locations in the contour plots.

Now that we have a representation of text data that is both continuous and computationally tractable, we can use our dimension reduced data for classification (e.g., determining the sentiment of a review via a generalized regression).

As there are many modeling options (any form of regression could be used), we opted for a GAM (generalized additive model) using spline smoothers (Wood 2017), as it doesn't assume linearity, produces an interpretable model (unlike a neural network), and can handle categorical data.

\footnotetext{
${ }^{1}$ As these dimensions have no straightforward interpretation, they are only labeled as X1 and X2. Despite being not interpretable by humans, they are still useful for prediction, as discussed later with the generalized adaptive model.
}
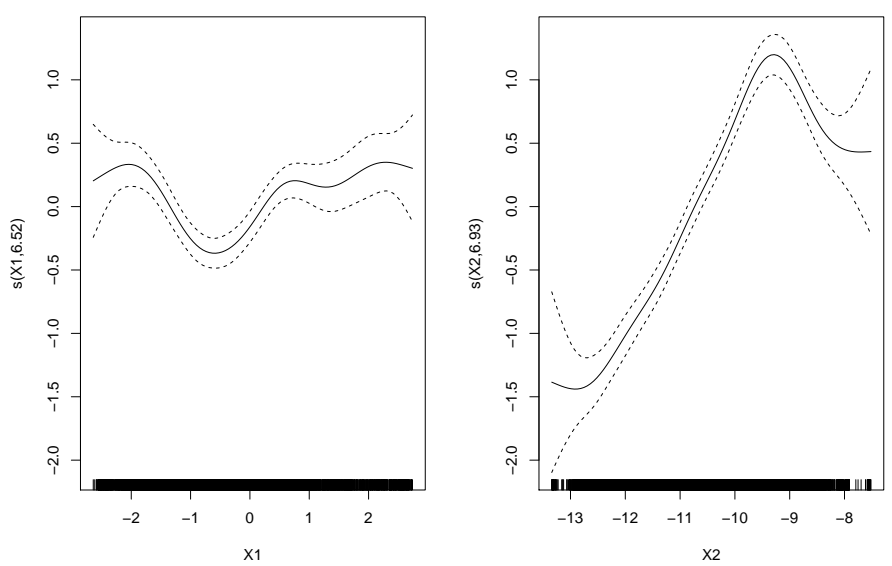

Figure 2: Using GAM and the dimension reduced data to predict movie sentiment.

\begin{tabular}{|c|c|c|c|}
\hline & P1 & P2 & P3 \\
\hline P1 & 0 & 1 & 0 \\
\hline P2 & 1 & 0 & 1 \\
\hline P3 & 0 & 1 & 0 \\
\hline
\end{tabular}

Table 1: An example of an adjacency matrix, depicting the friendship network between 3 people (P1, P2, and P3).

Since this is a statistical model, we can also present our model with new data and make predictions.

Model: sentiment $\sim s\left(X_{1}\right)+s\left(X_{2}\right)$

The resulting model has an R-square of 0.15 , which increases to approximately .20 at 4 dimensions, and at 10 dimensions has an R-square of about .22, so while there is an open question of how many dimensions should be extracted, we are clearly extracting useful data. The two non-linear components of GAM are visible in Figure 2. They depict the spline smoothed $X_{1}$ and $X_{2}$ dimensions.

\section{From text to networks}

As the UMAP procedure is so general we can also apply it to unusual kinds of data, like an adjacency matrix (Table 1) a way of representing a social network - by using Hamming distance as our distance measure. This means we can apply the technique to anonymize social network data.

As a proof of concept, here are two examples. When reduced to two dimensions, the well-known Zachary's karate club friendship network(Zachary 1977) results in almost perfect separation between the two club factions. Figure 3 depicts a scatter plot of the dimension reduced adjacency matrix, with a clear clustering of the two different factions, with only a single person misclustered.

A smaller though also famous network of marriage ties in Florence during the political competition between the Medici and Oligarch political factions (Padgett and Ansell 1993), results in a less clear picture in Figure 4 (likely due to the small number of nodes in the network). However, there is a strong relationship between having a X2 value greater than 2 and being a member of the Oligarch political faction. 


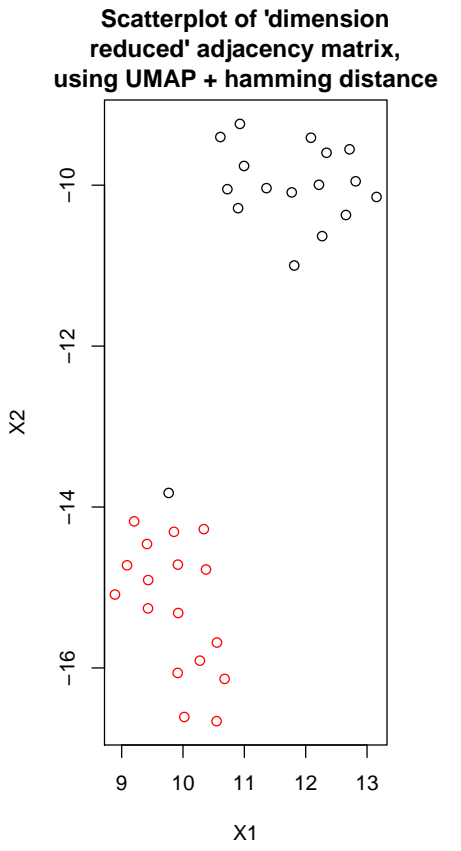

Figure 3: Comparison of the original social network with the dimension reduced scatter plot.

This relationship reveals the preservation of useful network data following the anonymization of the indiviual network member's ties, but it is no longer possible to identify which members have marriage ties, only clusters of members.

We can now convert both text and social network data into a reasonably anonymous format. Attempts to use the content of, say, a tweet, to identify an individual should be extremely difficult, as both the length and content of the tweet would be unknown, and we can combine this representation with network data that can't be easily deanonymized.

\section{Possible issues and future work}

By applying the UMAP procedure, we have now successfully anonymized both text and social network data in a form that can be used by researchers as part of a regression or other forms of analysis. This is the only known procedure capable of anonymizing social network data without making substantial and possibly misleading changes to the underlying network structure.

We are currently working on quantifying when the UMAP procedure extracts enough information from the original data to produce useful results. The procedure also cannot be applied to data where the original variables are the focus of a study. That is, we can't apply the procedure to age and income if we want to study how income is related to life span, as we could not disentangle the effect of age and income from one another. This hinders the procedures' applicability beyond text and social network data, as we often are interested in the relationships between, say, demographic variables.

Future work involves formally quantifying how difficult

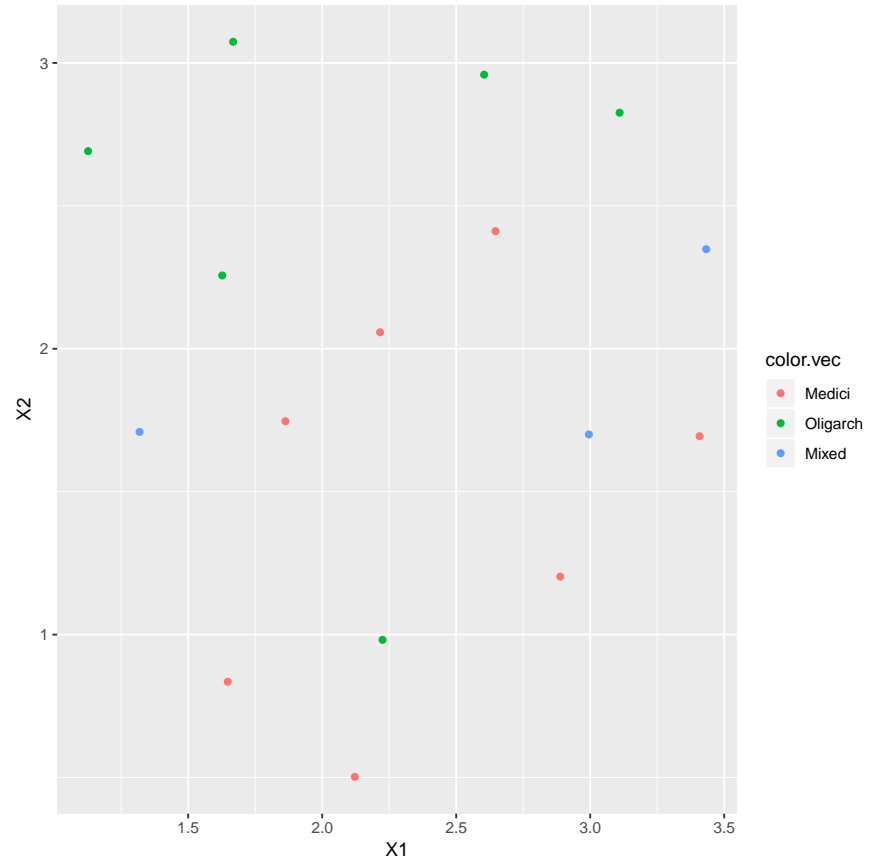

Figure 4: Comparison of the original social network with the dimension reduced scatter plot (Medici).

recovering the original data actually is. We also need to quantify just how reliable (and interpretable) the data from running the procedure on a social network actually is. Later work will also analyze the procedure's robustness via simulations.

\section{References}

Awodey, S. 2010. Category theory. Oxford University Press. Heffetz, O., and Ligett, K. 2014. Privacy and data-based research. Journal of Economic Perspectives 28(2):75-98.

McInnes, L., and Healy, J. 2018. Umap: Uniform manifold approximation and projection for dimension reduction. arXiv preprint arXiv:1802.03426.

Meyer, D.; Hornik, K.; and Feinerer, I. 2008. Text mining infrastructure in r. Journal of statistical software 25(5):154.

Narayanan, A., and Shmatikov, V. 2006. How to break anonymity of the netflix prize dataset. arXiv preprint cs/0610105.

Padgett, J. F., and Ansell, C. K. 1993. Robust action and the rise of the medici, 1400-1434. American journal of sociology 98(6):1259-1319.

Selivanov, D. 2016. text2vec: Modern text mining framework for r. Computer software manual]( $R$ package version 0.4. 0). Retrieved from https://CRAN. R-project. org/package $=$ text2vec .

Wood, S. N. 2017. Generalized additive models: an introduction with $R$. Chapman and Hall/CRC. 
Zachary, W. W. 1977. An information flow model for conflict and fission in small groups. Journal of anthropological research 33(4):452-473. 